\title{
Influence of ischemic injury on vein graft remodeling: Role of cyclic adenosine monophosphate second messenger pathway in enhanced vein graft preservation
}

Taichi Sakaguchi, MD, $\mathrm{PhD}^{\mathrm{a}}$
Tomohiro Asai, $\mathrm{MD}^{\mathrm{a}}$
Dmitri Belov, $\mathrm{MD}^{\mathrm{a}}$
Morihito Okada, MD, $\mathrm{PhD}^{\mathrm{a}}$
David J. Pinsky, MD
Ann Marie Schmidt, $\mathrm{MD}^{\mathrm{a}}$
Yoshifumi Naka, MD, $\mathrm{PhD}^{\mathrm{a}}$

From the Department of Surgery, ${ }^{\text {a }}$ College of Physicians and Surgeons, Columbia University, New York, NY, and the Department of Medicine, ${ }^{\text {b }}$ University of Michigan, Ann Arbor, Mich.

Current addresses of authors who have moved since this work was finished: Morihito Okada, The Hyogo Medical Center for Adults, Hyogo, Japan; David J. Pinsky, University of Michigan, Ann Arbor, Mich.

This study was supported by grants from the National Institutes of Health (KO8 HL04484 to Dr Naka, NIH grant HL60900 to Dr Pinsky) and from Uehara Memorial Foundation. Yoshifumi Naka is the Herbert Irving Assistant Professor of Surgery at Columbia University College of Physicians and Surgeons.

Received for publication Feb 1, 2004; revisions requested March 24, 2004; accepted for publication April 5, 2004.

Address for reprints: Yoshifumi Naka, MD, $\mathrm{PhD}$, Division of Cardiothoracic Surgery, Department of Surgery, New York Presbyterian Hospital, MHB 7-435, 177 Fort Washington Ave, New York, NY 10032 (E-mail: yn33@columbia.edu).

J Thorac Cardiovasc Surg 2005;129:129-37 $0022-5223 / \$ 30.00$

Copyright (c) 2005 by The American Association for Thoracic Surgery

doi:10.1016/j.jtcvs.2004.04.014
Objective: Endothelial injury during the harvest of saphenous vein grafts might play an important role in the development of vein graft disease after coronary artery bypass grafting. Using a murine autologous arterialized vein patch model, we tested whether the initial ischemic insult of vein grafts was linked to the later development of graft neointimal hyperplasia and whether the restoration of the cyclic adenosine monophosphate second messenger pathway would attenuate the development of neointimal hyperplasia.

Methods: A segment of the external jugular vein of a mouse was grafted onto its abdominal aorta. Three weeks after the operation, the degree of neointimal hyperplasia of the implanted graft was compared among (1) grafts without preservation, (2) grafts with 2 hours of preservation $\left(25^{\circ} \mathrm{C}\right)$ in heparinized saline, and (3) grafts with 2 hours of preservation in heparinized saline in the presence of a cyclic adenosine monophosphate analog. In addition, cyclic adenosine monophosphate contents of vein grafts and leukocyte adherence to the graft endothelium were assessed.

Results: Cyclic adenosine monophosphate contents were significantly decreased after 2 hours of preservation $(212 \pm 8$ vs $156 \pm 5 \mathrm{pmol} / \mathrm{L}, P<.01)$. The grafts preserved for 2 hours showed greater neointimal hyperplasia compared with the grafts without preservation (neointimal expansion, $68.7 \% \pm 9.6 \%$ vs $46.1 \% \pm 4.8 \% ; P<$ .01 ). The addition of a cyclic adenosine monophosphate analog to the preservation solution significantly suppressed neointimal hyperplasia of grafts preserved for 2 hours $(44.3 \% \pm 5.0 \%)$. Inhibiting the cyclic adenosine monophosphate-dependent protein kinase by adding Rp-cAMPS abrogated the beneficial effects. Furthermore, grafts preserved for 2 hours had significantly more leukocytes adhering to the graft endothelium 24 hours after the operation compared with nonpreserved grafts, which was significantly reduced by the cyclic adenosine monophosphate treatment.

Conclusions: Ischemic insult during vein graft harvest and preservation is a key factor in the development of vein graft neointimal hyperplasia at least in part caused by the depletion of cyclic adenosine monophosphate. We conclude that stimulation of the cyclic adenosine monophosphate second messenger pathway might be a potential strategy for the prevention of vein graft disease.

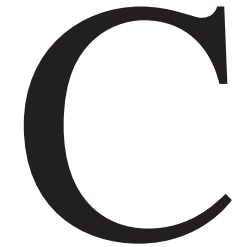

oronary artery bypass grafting is widely accepted for myocardial revascularization of patients with ischemic heart disease. In this setting the saphenous vein remains the most commonly used conduit, despite the increasing use of arterial grafts. However, the rate of saphenous vein graft failure remains high. Vein graft failure is comprised of 3 discrete processes: thrombosis, neointimal hyperplasia, and atherosclerosis. ${ }^{1-3}$ Various clinical strategies aimed at the prevention of 
vein graft failure have been used, including antiplateletanticoagulant drugs, risk factor modification, and lipidlowering drugs. However, most of these clinical trials have not addressed the problem of neointimal hyperplasia because the pathogenesis of this lesion is complex and remains obscure.

Endothelial dysfunction is thought to be an initial step in the development of neointimal hyperplasia. ${ }^{4}$ Mechanical or ischemic insults might enhance gene expression of adhesion molecules, growth factors, cytokines, and matrix proteins in endothelial cells, followed by smooth muscle cell migration-proliferation and accumulation of extracellular matrix..$^{1,2,5-8}$ Therefore maintenance of vascular integrity and function during the early period of vein graft implantation is one of the key factors linked to the subsequent development of neointimal hyperplasia. An important intracellular second messenger associated with endothelial cell regulation of vascular homeostatic properties is cyclic adenosine monophosphate (cAMP). ${ }^{9-12}$ Ischemia-driven cAMP deficiency in preserved organs is a critical mechanism by which vascular homeostasis might be disrupted. ${ }^{911-14}$ This study was designed to test the hypothesis that initial ischemic insult during harvest and storage of vein grafts is a central factor in the development of neointimal hyperplasia and that enhancement of graft preservation through supplementation of the cAMP pathway might attenuate vein graft neointimal hyperplasia.

\section{Methods}

\section{Mice and Vein Graft Procedure}

Male C57BL/6J mice were purchased from Jackson Laboratories. All procedures were approved by the Institutional Animal Care and Use Committee at Columbia University. The investigation conforms to the Guide for the Care and Use of Laboratory Animals published by the US National Institutes of Health (National Institutes of Health publication no. 85-23, revised 1996). The surgical procedures used were modified from those described by Shi and associates. ${ }^{15}$ Mice (12-16 weeks of age) were anesthetized by means of intraperitoneal injection of ketamine $(50 \mathrm{mg} / \mathrm{kg})$ and xylazine $(5 \mathrm{mg} / \mathrm{kg})$. A midline skin incision was made on the neck, and the right external jugular vein was dissected. A segment of the jugular vein ( $5 \mathrm{~mm}$ long) was transected after ligation at both ends with 8-0 sutures. This segment was opened longitudinally, washed with saline solution containing $100 \mathrm{U} / \mathrm{mL}$ heparin, and stored at $25^{\circ} \mathrm{C}$ for up to 2 hours in heparinized saline. After closing the neck incision with a 6-0 nylon suture, a midline skin incision was made on the abdomen. The abdominal aorta and the inferior vena cava were exposed. The segment of the abdominal aorta between the renal arteries and the aortic bifurcation was temporally occluded with a microvascular clamp (Roboz Surgical Instrument Co), and a longitudinal incision (of about the same length as the vein patch) was made. The defect of the aorta was repaired by suturing the prepared vein patch into the defect of the aorta with an 11-0 continuous suture around the margin of the patch. Contact between the instruments and the vein graft endothelium was avoided as much as possible throughout the procedure. After the vascular clamp was removed, the vein patch was inspected for adequacy of repair. The operation was considered successful if strong pulsation was confirmed in both the graft and native aorta without significant bleeding. If there were no pulsations or pulsations were diminished within a few minutes of restoration of blood flow, the procedure was considered a surgical failure. Cefazolin $(50 \mathrm{mg} / \mathrm{kg})$ was administered, and the skin incision was closed with a 6-0 nylon suture. Buprenorphine $(2.5 \mathrm{mg} / \mathrm{kg})$ was administered subcutaneously for postoperative analgesia. The duration of the entire procedure was approximately 50 minutes.

Figure 1 demonstrates the surgical procedure. The success rate of the operation exceeded $95 \%$. A total of 107 mice were included in this study. Ninety-three animals survived until their designated time of harvest. Two mice died intraoperatively from complications caused by anesthesia, and 12 mice died postoperatively as a result of ileus $(n=3)$, intra-abdominal bleeding $(n=2)$, graft occlusion $(n=2)$, or unknown cause $(n=5)$. The overall success rate was $87 \%$.

\section{Graft Preservation}

The base preservation solution consisted of saline with heparin $(100 \mathrm{U} / \mathrm{mL})$ alone or with the following additional reagents: $N^{6}, 2^{\prime}-$ $O$-dibutyryl adenosine 3':5'-cyclic monophosphate (db-cAMP; 2 $\mathrm{mmol} / \mathrm{L})$; 8-bromoadenosine-cAMP (8-Br-cAMP; $0.1 \mathrm{mmol} / \mathrm{L}$ ); and 8 -Br-cAMP $(0.1 \mathrm{mmol} / \mathrm{L})$ plus Rp-cAMPS $(0.25 \mathrm{mmol} / \mathrm{L})$, the Rp isomer of adenosine 3',5'-monophosphate (Sigma). ${ }^{16}$ The concentrations of these cAMP analogues have been shown to be the optimal dose on the basis of previous studies, ${ }^{12,13,16}$ and adding cAMP analogues did not change the acidity of the base preservation solution.

\section{Morphology}

Mice were killed at various time points after surgical intervention and perfusion fixed with $10 \%$ formaldehyde at physiologic pressure. The grafts, together with a short segment of the native abdominal artery, were harvested and cut at the center. The specimens were embedded in medium (OCT compound) and frozen at $-80^{\circ} \mathrm{C}$. The section $(5 \mu \mathrm{m})$ at the midportion of each composite graft was stained with hematoxylin and eosin or elastic van Gieson stain (Sigma), and the degree of neointimal expansion was analyzed quantitatively with a Zeiss microscope and image analysis system (Media Cybernetics). The consistency of the neointimal formation in the central portion of the graft was histologically confirmed by analyzing serial sections from the center to the proximal and distal ends of the graft (data not shown). ${ }^{15}$ The neointima of the vein graft was defined as the region between the lumen and the adventitia. Neointimal cell number was calculated by counting the number of nuclei visible in sections stained with hematoxylin and eosin. The percentage of neointimal expansion was calculated as follows: $100 \times($ Neointimal area/Neointimal area + Luminal area). These quantifications were performed by an observer blinded to the experimental circumstances. Masson trichrome staining was performed according to the manufacturer's instruction (Sigma).

\section{cAMP Assay}

Segments of inferior vena cava were taken without $(n=4)$ or with 2 hours of preservation in the heparinized saline solution $\left(25^{\circ} \mathrm{C}, \mathrm{n}\right.$ 

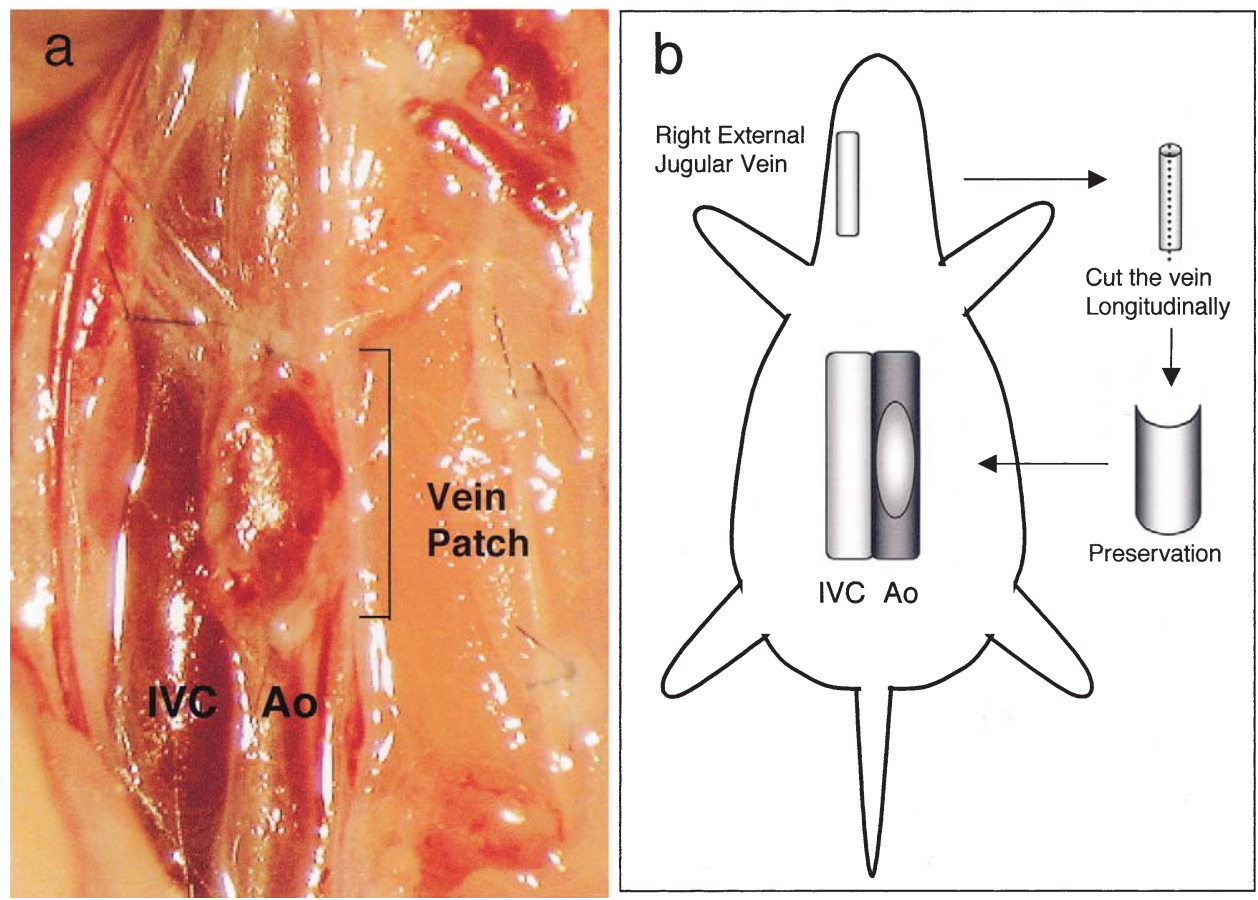

Figure 1. Photograph (a) and schematic representation (b) demonstrating the procedures of the vein patch implantation model. A 5-mm-long vein segment taken from the right external jugular vein was sutured onto the abdominal aorta after preservation. IVC, Inferior vena cava; Ao, aorta.

$=4)$, snap-frozen in liquid nitrogen, and stored at $-80^{\circ} \mathrm{C}$. Tissue samples were ground to a fine powder under liquid nitrogen, weighed, and homogenized in 10 volumes of $0.1 \mathrm{~N} \mathrm{HCl}$. cAMP immunoassay was performed according to the manufacturer's instruction (R\&D Systems Inc).

\section{En Face Immunofluorescence}

The procedure used in this study was similar to that reported by Zou and colleagues ${ }^{17}$ and Dietrich and coworkers. ${ }^{18}$ Vein patch was retrieved 24 hours after the operation, mounted on a glass slide with the endothelium side up, and air-dried for 1 hour at room temperature. The segments were fixed in cold acetone $\left(-20^{\circ} \mathrm{C}\right)$ for 10 minutes and rinsed in phosphate-buffered saline. The segments were then incubated with rat monoclonal antibody to macrophage-1 antigen (MAC-1, CD116/CD18) (1:25, Pharmingen) for 30 minutes and visualized with fluorescein isothiocyanate-labeled rabbit anti-rat $\operatorname{IgG}$ (1:25, Sigma). MAC-1-positive cells were blindly counted at $400 \times$ magnification in 10 fields of each segment.

\section{Immunohistochemistry}

Representative sections $(5 \mu \mathrm{m})$ were immunostained with rat anti-platelet endothelial cell adhesion molecule-1 (anti-PECAM-1, anti-CD31) antibody (1:100, Pharmingen), rat anti-MAC-1 antibody (1:50, Pharmingen), and hamster anti-intercellular adhesion molecule 1 (anti-ICAM-1) antibody (1:100, Pharmingen). Sections were blocked with hydrogen peroxide $(0.3 \%)$ in methanol for 10 minutes. Blocking was performed with goat serum $(4 \%)$ and bovine serum albumin $(1 \%)$ in phosphate-buffered saline. Primary antibodies were added to slides and incubated overnight at $4^{\circ} \mathrm{C}$. Secondary antibodies (1:100; anti-hamster or anti-rat IgG, Pharmingen) were added for 30 minutes at room temperature. Sections were reacted with horseradish peroxidase-conjugated streptavidin (1:100, Sigma) for 30 minutes at room temperature and developed with 3.3'-diaminobenzidine (DAB substrate kit, Vector).

\section{Statistical Analysis}

All data are expressed as means \pm SEM. The Student unpaired $t$ test for a comparison between 2 groups or analysis of variance with post hoc analysis using the Bonferroni-Dunn test for a comparison among more than 2 groups was used to determine significant difference. All analyses were performed by using the Statview statistical package, version J5.0 (Abacus Concepts Inc).

\section{Results}

\section{Neointimal Formation in Jugular Vein Autograft}

Figure 2 shows the representative histologic sections of vein patch grafts that had been implanted onto the abdominal aorta immediately after harvest without preservation. Elastic van Gieson stain (Figure 2, a) showed that neointimal hyperplasia developed in the vein patch graft 21 days after the operation. The neointima formed primarily in association with the venous part of the composite vessel (black arrows) and not the arterial part, which is easily distinguished from the venous part by the existence of the elastic laminas (stained as black). Masson trichrome stain (Figure 

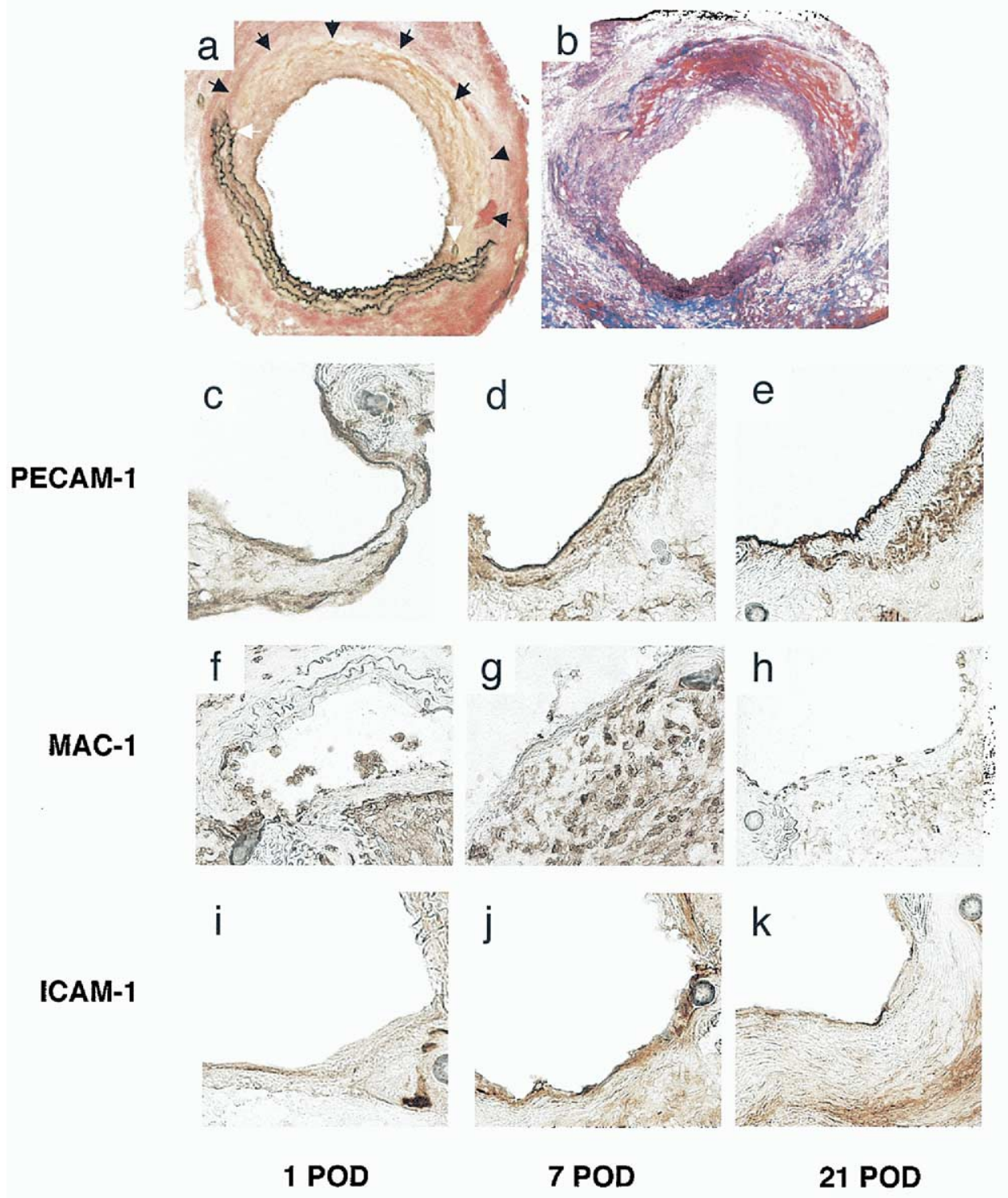

Figure 2. Photomicrographs of cross-sections of jugular vein-abdominal aorta composite vessels. Sections are oriented with the vein patch on top and the artery segment on bottom. Elastic van Gieson stain for postoperative 21-day samples shows elastic lamina of the arterial wall as black, muscle as yellow, and collagen as red (a). White arrows indicate sutures approximately corresponding to the junction of the vein patch (black arrows) and arterial wall. Masson trichrome stain for postoperative 21-day samples shows the extracellular matrix as blue and the cellular portion as red (b). Immunohistologic staining for PECAM-1 (CD31; c-e), MAC-1 (f-h), and ICAM-1 (i-k) for postoperative days 1 (c, f, and i), 7 (d, g, and j), and 21 (e, h, and $k$ ) is also shown. (Original magnification 100x [a and b] or $400 \times(c-k)$.

$2, b)$ indicated that the bulk of the lesion was formed by collagen (blue) and smooth muscle cells (red), which were visible by means of $\alpha$-actin staining (not shown). PECAM-1 (CD31) staining indicates some varieties of continuity of the endothelium in the venous part at day 1 (Figure 2,c), which became more continuous at days 7 and 21 (Figure 2, $d$ and e). $\mathrm{MAC}-1^{+}(\mathrm{CD} 11 \mathrm{~b} / 18)$ monocytes-macrophages were detected at the luminal surface at 1 day after the operation and were seen transmurally by 3 weeks (Figure $2, f-h$ ). MAC- $1^{+}$ cells were predominant in the neointima of the vein graft at postoperative day 7 and decreased their fraction in the neointima at postoperative day 21 , when they were replaced 

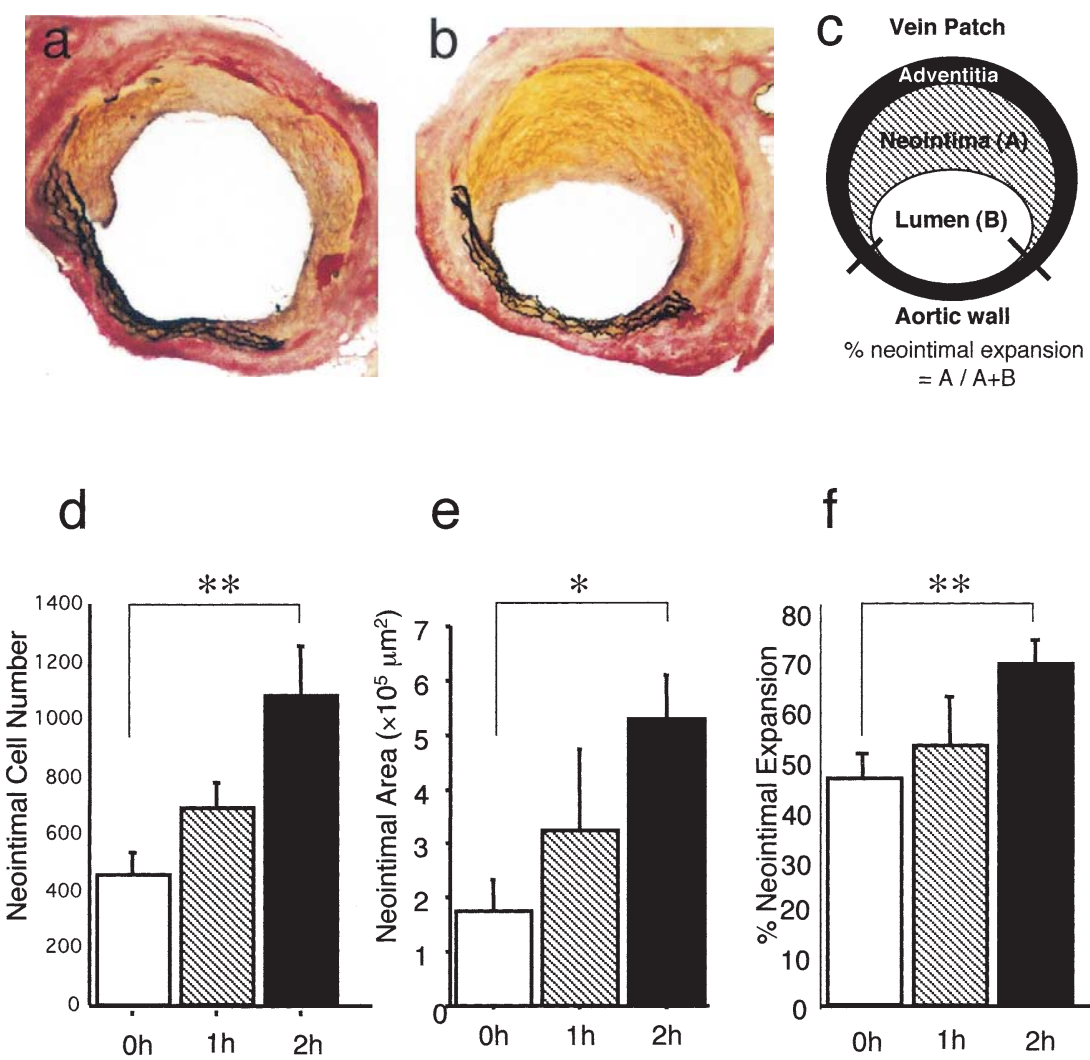

\section{e}

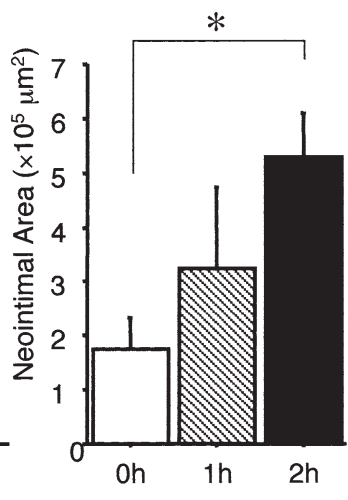

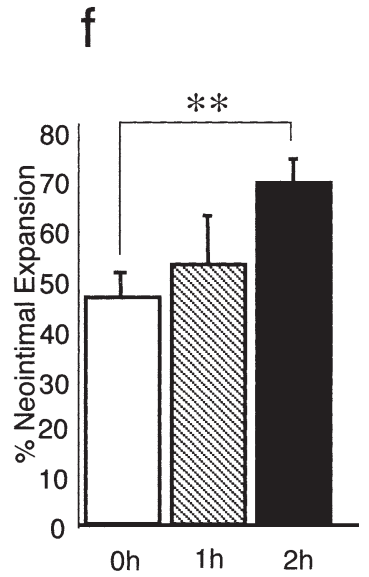

Figure 3. $a$ and $b$, Influence of perioperative ischemic injury on the development of neointimal hyperplasia of vein grafts. Elastic van Gieson-stained sections of jugular vein-abdominal aorta composite vessels 21 days after the operation are shown: a, vein graft that had been implanted immediately after harvest, without an intervening preservation period; $\mathbf{b}$, vein graft subjected to 2 hours of preservation in heparinized saline before implantation. (Original magnification $100 \times$.) c, Schematic representation of a cross-section of the composite vessel. Statistical data of the total neointimal cell number (d), neointimal area (e), and percentage of neointimal expansion (f) at postoperative day 21 are shown as means \pm SEM for each group. Numbers of grafts were as follows: 15 in the nonpreservation group, 5 in the 1-hour preservation group, and 9 in the 2-hour preservation group. ${ }^{*} \boldsymbol{P}<.05,{ }^{*} \boldsymbol{P}$ $<.01$.

by smooth muscle cells. Expression of ICAM-1, a counterreceptor for MAC-1, in the vein graft significantly increased 1 day after implantation and continued to be increased at postoperative days 7 and 21 (Figure 2, $i-k$ ).

\section{Accelerated Neointimal Formation in Jugular Vein Autograft by Ischemic Preservation}

Figure 3 shows elastic van Gieson-stained micrographs of a section of the jugular vein-abdominal aorta composite vessels 21 days after the operation (Figure 3, $a$ and $b$ ) and statistical data in terms of neointimal cell number (Figure 3, $d$ ), neointimal area (Figure 3,e), and percentage of neointimal expansion (Figure 3, f), which was calculated as described (Figure 3,c). Even without preservation, implanted vein grafts showed neointimal formation 3 weeks after the operation. When vein grafts were subjected to 2 hours of preservation, they exhibited a significant increase in the severity of neointimal hyperplasia in terms of neointimal cell number (Figure 3, $d ; 454 \pm 77$ in nonpreserved grafts vs $1081 \pm 173$ cells/neointimal area in 2-hour preserved grafts; $P<.01$ ), neointimal area (Figure 3, $e ; 1.759$ \pm 0.584 vs $5.269 \pm 0.820 \times 10^{5} \mu \mathrm{m}^{2}$, respectively; $P<$ $.05)$, and neointimal expansion ( $46.1 \% \pm 4.8 \%$ vs $68.7 \% \pm$ $9.6 \%$, respectively; $P<.01$ ).

\section{cAMP contents}

Compared with no preservation, 2 hours of preservation was associated with significantly decreased cAMP content (212 \pm 8 vs $156 \pm 5 \mathrm{pmol} / \mathrm{L}$, respectively; $P<.01$; Figure $4, a$ ).

\section{Reduced Neointimal Formation by cAMP Treatment}

Figure $4, b$ shows the effects of cAMP treatment during 2 hours of preservation on the development of neointimal expansion of vein grafts 21 days after the operation. Adding 
a

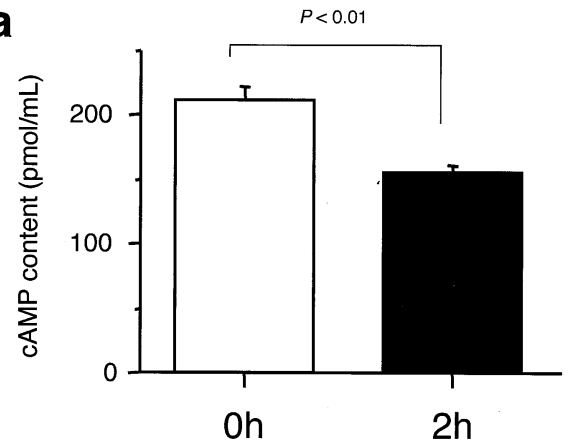

b

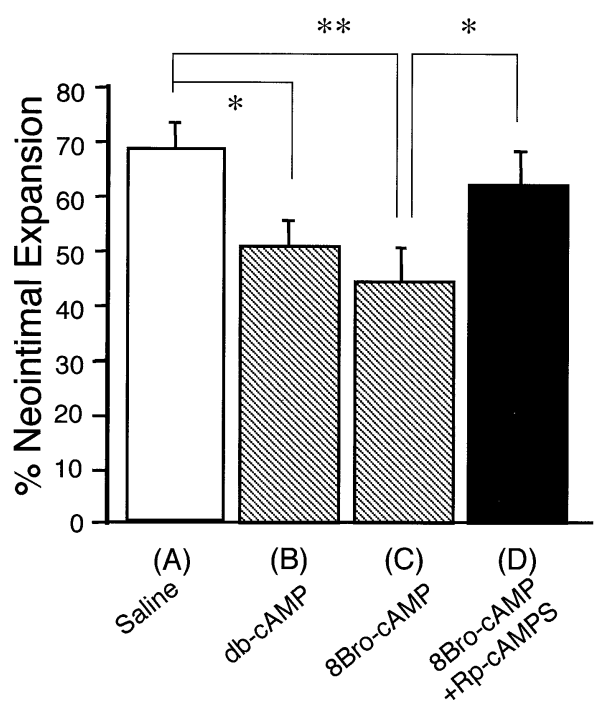

Figure 4. a, cAMP contents in the harvested vein graft. b, Effect of cAMP analogues (db-cAMP and 8-Br-cAMP) on neointimal expansion of vein grafts. The vein patch was stored in heparinized saline without any additives (A); with cAMP analog $d b$ CAMP (2 mmol/L; B), 8-Br-CAMP (0.1 mmol/L; C), or 8-Br-cAMP (0.1 $\mathrm{mmol} / \mathrm{L})$; and with the CAMP-dependent protein kinase inhibitor Rp-cAMPS ( $0.25 \mathrm{mmol} / \mathrm{L} ; \mathrm{D})$. After 2 hours of preservation, the vein patch was implanted onto the abdominal aorta of the same mouse and harvested at postoperative day 21. Data are shown as means \pm SEM for each group. Numbers of grafts were as follows: $A, 9$; B, 9; C, 9; D, 8. * $P<.05,{ }^{* *} P<.01$.

the membrane-permeable cAMP analog db-cAMP (2 $\mathrm{mmol} / \mathrm{L}$ ) to the preservation solution resulted in markedly diminished neointimal formation at 3 weeks (neointimal expansion is $68.7 \% \pm 9.6 \%$ without additives $[A]$ vs $51.0 \% \pm 4.7 \%$ with db-cAMP $[B], P<.05)$. To demonstrate that the beneficial effects of db-cAMP were not specific for this cAMP analog and accrue through activation by cAMP of the cAMP-dependent protein kinase, an additional series of experiments was performed. Incorporation of the membrane-permeable cAMP analog 8-Br-cAMP (0.1 $\mathrm{mmol} / \mathrm{L}$ ) into the preservation solution caused a similar reduction of neointimal formation at 3 weeks $(44.3 \% \pm$
$5.0 \%[C]$ vs without additives $[A], P<.01)$. When the same dose of 8 -Br-cAMP was added to the preservation solution in the presence of additional cAMP-dependent protein kinase inhibitor, Rp-cAMPS $(0.25 \mathrm{mmol} / \mathrm{L})$, the beneficial effects of the cAMP analog with respect to diminishing neointimal formation were abolished $(62.1 \% \pm 6.2 \%[D]$ vs with 8 -Br-cAMP only $[C], P<.01)$.

\section{Leukocyte Adhesion-Infiltration to Vein Grafts}

Cells adhering to the endothelial surface were visualized with a rat monoclonal antibody recognizing $\mathrm{MAC}-1^{+}$leukocytes (CD11b/CD18; Figure 5, $a-c)$. Significant numbers of MAC- $1^{+}$leukocytes (ie, monocytes-macrophages) were observed adherent to the surface of nonpreserved vein graft segments a day after the operation (Figure 5,a). The number of $\mathrm{MAC}-1^{+}$leukocytes was significantly greater in grafts exposed to 2 hours of preservation than in nonpreserved vein grafts $(P<.05$; Figure 5, $b$ and $d)$. Furthermore, adding db-cAMP to the preservation solution resulted in markedly decreased $\mathrm{MAC}-1^{+}$cells in the vein grafts preserved for 2 hours $(P<.05$; Figure 5, $c$ and $d)$. Immunostaining for MAC-1 in the grafts harvested on day 21 showed that more MAC- $1^{+}$cells were detected in the neointima of 2-hour preserved grafts (Figure 5, f) compared with those in nonpreserved grafts (Figure 5,e) and 2-hour preserved grafts treated with cAMP (Figure 5, g). These results indicate that leukocyte adhesion to the endothelium is one of the earliest cellular events in vein graft disease, and the beneficial effect of cAMP on preventing vein graft disease was partly due to inhibition of leukocyte adhesion.

\section{Discussion}

Early endothelial cell injury has been focused on as a key factor contributing to the later development of vein graft failure. ${ }^{4,19-21}$ Damage to endothelial cells is caused by several factors, including mechanical manipulation during harvest, ${ }^{6,19}$ ischemia during graft preservation, ${ }^{5,10,22}$ and increased shear stress caused by higher arterial blood pressure once implanted..$^{8,23-25}$ These factors might stimulate endothelial cells to release inflammatory mediators and growth factors with suppression of the anticoagulant cofactors, resulting in the later development of intimal hyperplasia. ${ }^{1,3,4}$ In this study we focused on ischemia-induced endothelial dysfunction. Previous histochemical analyses have suggested that structural derangements in the harvested veins can be detected within 2 hours of storage. ${ }^{4,22}$ The level of active extracellular signal-regulated kinase in human saphenous vein is markedly increased after 50 minutes of incubation in normal saline solution. ${ }^{7}$ Recently, Tatte and associates $^{22}$ assessed the structural and functional integrity of human saphenous vein segments stored in multiple preservation solutions using multiphoton microscopy. They showed that within 60 minutes of harvest and storage in 

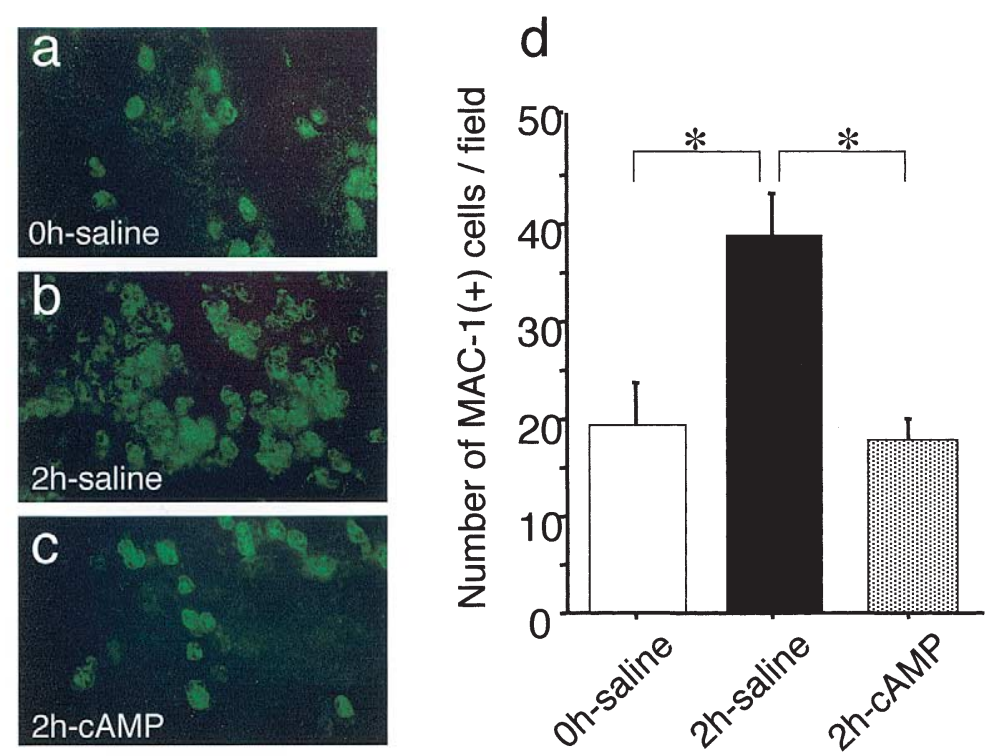

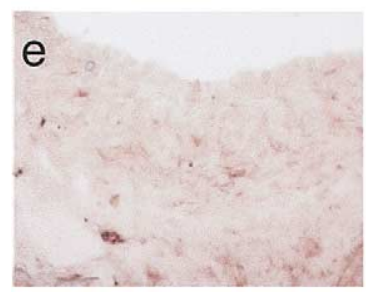

Oh-saline

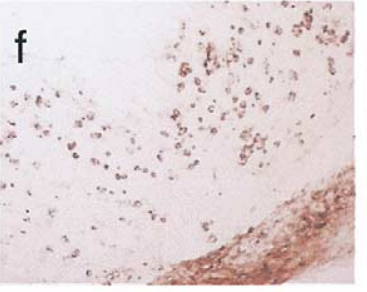

2h-saline

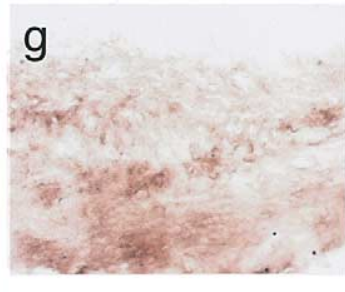

2h-cAMP

Figure 5. Leukocyte adhesion-infiltration to the vein patch. En face immunofluorescence demonstrated MAC-1 ${ }^{+}$ cells adhering to the endothelium of the vein patch (a-c): $A$, vein graft without preservation; $b$, vein grafts subjected to 2 hours of preservation before implantation without preservation solution additive; c, vein grafts subjected to 2 hours of preservation with added cAMP analog db-cAMP. (Original magnification $200 \times$.) d, MAC-1 ${ }^{+}$cells adhered to the surface of the vein patch. Data are shown as means \pm SEM for each group $(n=10)$. Immunohistochemical staining demonstrates the presence of $\mathrm{MAC}-1^{+}$cell infiltration in vein grafts at postoperative day 21 (e-g). (Original magnification $400 \times$.)

heparinized lidocaine saline or autologous heparinized blood, calcium mobilization and nitric oxide generation were markedly diminished, with more than $90 \%$ of endothelial cells no longer viable in the vein. In this study vein grafts preserved in heparinized saline for 2 hours showed significantly decreased tissue cAMP contents and significantly increased leukocyte adherence, followed by a marked increase in neointimal expansion 21 days after the operation compared with that seen in nonpreserved grafts. These results indicate that early endothelial damage caused by ischemic preservation might be an important contributor to the later vein graft remodeling.

cAMP is known to be an important intracellular second messenger associated with endothelial cell regulation of vascular homeostatic properties. Previous studies have shown that stimulation of the cAMP pathway prevents neointimal formation after balloon injury of the rat carotid artery and proliferation of vascular smooth muscle cells in vitro. ${ }^{26-28}$ cAMP also inhibits tumor necrosis factor $\alpha$-induced release of IL- 6 and migration of vascular smooth muscle cells cultured from human saphenous vein. ${ }^{14}$ In porcine saphenous vein and carotid artery interposition grafts, cAMP-synthesizing capacity is significantly downregulated at 1 month after implantation, which might be relevant to the pathophysiology of early vein graft failure. ${ }^{29,30}$ A recent report of the 5-year interim results of a randomized clinical trial of graft patency after coronary artery bypass surgery showed high patency of saphenous vein grafts (94\%), speculating that bathing vein grafts in phosphodiesterase III inhibitor before implantation is one of the causes associated with the very low rate of graft failure. ${ }^{31}$ Furthermore, we have previously reported that in 
murine cardiac allografts the restoration of the cAMP pathway at the time of preservation not only improved acute allograft function but also reduced the severity of transplant-associated coronary artery disease in grafts examined 2 months after surgical intervention. ${ }^{16}$ The donor hearts preserved for 120 minutes in the presence of db-cAMP showed significantly more nitric oxide production and less superoxide release, followed by less neointimal hyperplasia 60 days after transplantation, compared with that seen in the control hearts preserved without the cAMP analog. In this study, on the basis of the finding that tissue cAMP contents were significantly decreased after 2 hours of preservation, we hypothesized that the restoration of the cAMP pathway would enhance vein graft preservation and thus attenuation of the later development of neointimal hyperplasia.

cAMP regulates induction of a distinct set of genes, including $T N F A, I L 6,{ }^{14} T F$, the gene encoding E-selectin, $V C A M 1,{ }^{32,33}$ and $I C A M 1 .{ }^{34}$ Adhesion molecules play an important role in leukocyte adhesion to the endothelium, which is a primary step in the early stage of atherosclerosis. ${ }^{35}$ Several lines of evidence have suggested that ICAM1/MAC-1-dependent cellular interaction is involved in a number of inflammatory processes and in atherosclerosis through mononuclear cell adhesion and migration. ${ }^{17,18,36,37}$ Zou and associates ${ }^{17}$ have shown that neointimal hyperplasia of vein bypass grafts was significantly reduced in ICAM-1-deficient mice compared with in wild-type animals. This is consistent with our preliminary study (data not shown). In this study MAC- $1^{+}$cells adhering to the vein graft surface were significantly increased by 2 hours of preservation, and cAMP treatment markedly suppressed leukocyte adherence. In addition, ICAM-1 expression of the graft surface was increased by ischemic preservation (data not shown). Therefore inhibition of leukocyte adhesion by reduction of ICAM-1 expression might be one of the important underlying mechanisms by which cAMP treatment suppressed neointimal expansion of vein grafts.

Several animal models have been developed to clarify the underlying mechanism of vein graft remodeling. Murine genetic models have considerable advantages over other animal systems in that they overcome the need to administer factors or their inhibitors. Zou and associates ${ }^{17,38,39}$ established the first murine model of vein graft arteriosclerosis wherein the external jugular vein or inferior vena cava were autografted-isografted into carotid arteries by using the cuff technique. Shi and coworkers ${ }^{15}$ also reported another murine model in which a patch cut from the external jugular vein was grafted to repair a surgically created defect in the carotid artery. In the current study we used a murine vein patch implantation model with some modification of that reported by the latter. The vein patch taken from the external jugular vein was implanted onto the abdominal aorta of the same animal. This method is technically easier than that of Shi and coworkers ${ }^{15}$ and provides a means to avoid technical problems of interposition caused by size mismatch of the vein and artery. We believe that this model represents a significant advance toward understanding the pathogenesis and treatment of vein graft disease.

In summary, our data indicate that initial ischemic insult plays an important role in the later development of vein graft neointimal hyperplasia and that stimulation of the cAMP second messenger pathway at the time of graft harvest might be a potential strategy for the prevention of vein graft disease.

\section{References}

1. Davies MG, Hagen PO. Pathophysiology of vein graft failure: a review. Eur J Vasc Endovasc Surg. 1995;9:7-18.

2. Motwani JG, Topol EJ. Aortocoronary saphenous vein graft disease: pathogenesis, predisposition, and prevention. Circulation. 1998;97: 916-31.

3. Mehta D, Izzat MB, Bryan AJ, Angelini GD. Towards the prevention of vein graft failure. Int J Cardiol. 1997;62(suppl 1):S55-63.

4. Thatte HS, Khuri SF. The coronary artery bypass conduit: I. Intraoperative endothelial injury and its implication on graft patency. Ann Thorac Surg. 2001;72(suppl):S2245-52.

5. Michiels C, De Leener F, Arnould T, Dieu M, Remacle J. Hypoxia stimulates human endothelial cells to release smooth muscle cell mitogens: role of prostaglandins and bFGF. Exp Cell Res. 1994;213: 43-54.

6. Souza DSR, Dashwood MR, Tsui JCS, Filbey D, Bodin L, Johansson $\mathrm{B}$, et al. Improved patency in vein grafts harvested with surrounding tissue: results of a randomized study using three harvesting techniques. Ann Thorac Surg. 2002;73:1189-95.

7. Bizekis MD, Pintucci G, Derivaux CC, Saponara F, Kim JH, Hyman $\mathrm{KM}$, et al. Activation of mitogen-activated protein kinases during preparation of vein grafts and modulation by a synthetic inhibitor. J Thorac Cardiovasc Surg. 2003;126:659-65.

8. Chello M, Mastroroberto P, Frati G, Patti G, D'Ambrosio A, Sciascio GD, et al. Pressure distention stimulates the expression of endothelial adhesion molecules in the human saphenous vein graft. Ann Thorac Surg. 2003;76:453-8.

9. Pinsky DJ, Yan SF, Lawson C, Naka Y, Chen JX, Connolly ES Jr, et al. Hypoxia and modification of the endothelium: implications for regulation of vascular homeostatic properties. Semin Cell Biol. 1995; 6:283-94.

10. Michiels C, Arnould T, Remacle J. Endothelial cell responses to hypoxia: initiation of a cascade of cellular interactions. Biochim Biophys Acta. 2000;1497:1-10.

11. Ogawa S, Koga S, Kuwabara K, Brett J, Morrow B, Morris SA, et al. Hypoxia-induced increased permeability of endothelial monolayers occurs through lowering of cellular cAMP levels. Am J Physiol. 1992;262:C546-54.

12. Naka Y, Roy DK, Liao H, Chowdhury NC, Michler RE, Oz MC, et al. cAMP-mediated vascular protection in an orthotopic rat lung transplant model. Insights into the mechanism of action of prostaglandin E1 to improve lung preservation. Circ Res. 1996;79:773-83.

13. Kayano K, Toda K, Naka Y, Okada K, Oz MC, Pinsky DJ. Superior protection in orthotopic rat lung transplantation with cyclic adenosine monophosphate and nitroglycerin-containing preservation solution. J Thorac Cardiovasc Surg. 1999;118:135-44.

14. Newman WH, Castresana MR, Webb JG, Wang Z. Cyclic AMP inhibits production of interleukin-6 and migration in human vascular smooth muscle cells. J Surg Res. 2003;109:57-61.

15. Shi C, Patel A, Zhang D, Wang H, Carmeliet P, Reed GL, et al. Plasminogen is not required for neointima formation in a mouse model of vein graft stenosis. Circ Res. 1999;84:883-90.

16. Wang CY, Aronson I, Takuma S, Homma S, Naka Y, Alshafie T, et al. 
cAMP pulse during preservation inhibits the late development of cardiac isograft and allograft vasculopathy. Circ Res. 2000;86:982-98.

17. Zou Y, Hu Y, Mayr M, Dietrich H, Wick G, Xu Q. Reduced neointima hyperplasia of vein bypass grafts in intercellular adhesion molecule1-deficient mice. Circ Res. 2000;86:434-40.

18. Dietrich H, Hu Y, Zou Y, Dirnhofer S, Kleindienst R, Wick G, et al. Mouse model of transplant arteriosclerosis: role of intercellular adhesion molecule-1. Arterioscler Thromb Vasc Biol. 2000;20:343-52.

19. Davies MG, Dalen H, Svendsen E, Hagen PO. Influence of perioperative catheter injury on the long-term vein graft function and morphology. J Surg Res. 1996;66:109-14.

20. Fulton GJ, Davies MG, Barber L, Gray JL, Svendsen E, Hagen PO. Local effects of nitric oxide supplementation and suppression in the development of intimal hyperplasia in experimental vein grafts. Eur $J$ Vasc Endovasc Surg. 1998;15:279-89.

21. Cavallari N, Abebe W, Mingoli A, Sapienza P, Hunter WJ, Agrawal DK, et al. Short-term preservation of autogenous vein grafts: effectiveness of University of Wisconsin solution. Surgery. 1997;121:6471.

22. Tatte S, Biswas KS, Najjar SF, Birjiniuk V, Crittenden MD, Michel T, et al. Multi-photon microscopic evaluation of saphenous vein endothelium and its preservation with a new solution, GALA. Ann Thorac Surg. 2003;75:1145-52.

23. Golledge J, Turner RJ, Harley SL, Springall DR, Powell JT. Circumferential deformation and shear stress induce differential responses in saphenous vein endothelium exposed to arterial flow. J Clin Invest. 1997;99:2719-26.

24. Gosling M, Golledge J, Turner RJ, Powell JT. Arterial flow conditions downregulate thrombomodulin on saphenous vein endothelium. Circulation. 1999;99:1047-53.

25. Jeremy JY, Dashwood MR, Mehta D, Izzat MB, Shukla N, Angelini GD. Nitric oxide, prostacyclin and cyclic nucleotide formation in externally stented porcine vein grafts. Atherosclerosis. 1998;141:297305.

26. Indolfi C, Di Lorenzo E, Rapacciuolo A, Stingone AM, Stabile E, Leccia A, et al. 8-chloro-cAMP inhibits smooth muscle cell proliferation in vitro and neointima formation induced by balloon injury in vivo. J Am Coll Cardiol. 2000;36:288-93.

27. Indolfi C, Stabile E, Coppola C, Gallo A, Perrino C, Allevato G, et al. Membrane-bound protein kinase A inhibits smooth muscle cell pro- liferation in vitro and in vivo by amplifying cAMP-protein kinase A signals. Circ Res. 2001;88:319-24.

28. Bornfeldt KE, Krebs EG. Crosstalk between protein kinase A and growth factor receptor signaling pathways in arterial smooth muscle. Cell Signal. 1999;11:465-77.

29. Jeremy JY, Birkett SD, Bryan AJ, Angelini GD. The influence of surgical preparation on cyclic nucleotide synthesis in an organ culture of human saphenous vein. Eur J Vasc Endovasc Surg. 1997;13:72-8.

30. Jeremy JY, Dashwood MR, Timm M, Izzat MB, Mehta D, Bryan AJ, et al. Nitric oxide synthase and adenylyl and guanylyl cyclase activity in porcine interposition vein grafts. Ann Thorac Surg. 1997;63:470-6.

31. Buxton BF, Raman JS, Ruengsakulrach P, Gordon I, Rosalion A, Bellomo R, et al. Radial artery patency and clinical outcomes: fiveyear interim results of a randomized trial. J Thorac Cardiovasc Surg. 2003;125:1363-71.

32. Ollivier V, Parry GC, Cobb RR, de Prost D, Mackman N. Elevated cyclic AMP inhibits NF-kappaB-mediated transcription in human monocytic cells and endothelial cells. J Biol Chem. 1996;271:2028-35.

33. Lalli E, Sassone-Corsi P. Signal transduction and gene regulation: the nuclear response to cAMP. J Biol Chem. 1994;269:1759-62.

34. Balyasnikova IV, Pelligrino DA, Greenwood J, Adamson P, Dragon S, Raza H, et al. Cyclic adenosine monophosphate regulates the expression of the intercellular adhesion molecule and the inducible nitric oxide synthase in brain endothelial cells. J Cereb Blood Flow Metab. 2000;20:688-99.

35. Stark VK, Hoch JR, Warner TF, Hullett DA. Monocyte chemotactic protein-1 expression is associated with the development of vein graft intimal hyperplasia. Arterioscler Thromb Vasc Biol. 1997;17:1614-21.

36. Crook MF, Newby AC, Southgate KM. Expression of intercellular adhesion molecules in human saphenous veins: effects of inflammatory cytokines and neointima formation in culture. Atherosclerosis. 2000;150:33-41.

37. Vural KM, Oz MC, Liao H, Batirel HF, Pinsky DJ. Membrane stabilization in harvested vein graft storage: effects on adhesion molecule expression and nitric oxide synthesis. Eur J Cardiothorac Surg. 1999; $16: 150-5$.

38. Dietrich H, Hu Y, Zou Y, Huemer U, Metzler B, Li C, et al. Rapid development of vein graft atheroma in ApoE-deficient mice. Am J Pathol. 2000;157:659-69.

39. Zou Y, Dietrich H, Hu Y, Metzler B, Wick G, Xu Q. Mouse model of venous bypass graft arteriosclerosis. Am J Pathol. 1998;153:1301-10. 